\title{
Bariatric Surgery: preoperative assessment
}

\author{
Hawa Juma El-Shareif \\ Department of Endocrine, Tripoli, Medical Center, Faculty of \\ Medicine, Tripoli University for Medical Sciences, Tripoli, Libya \\ E-mail: h.el_shareif@uot.edu.ly
}

\begin{abstract}
Obesity is associated with increased morbidity and mortality and impaired quality of life. Weight losse is associated with improvement or even cure of cardiovascular risk factors with cardiometabolic benefit. nonsurgical measures for weight loss is rarely successful in the long term. Bariatric surgery has achieved clear success in reversing the abnormal metabolic profile associated with obesity. Pre surgical evaluation is important to rule out any medical or psychological problems that increased the surgical mortality or adversely affect the outcome.
\end{abstract}

$\underline{\text { Keywords: }}$ obesity; bariatric surgery; preoperative evaluation

\section{Introduction}

Obesity is one of the most important public health challenges of the present century; its prevalence is increasing rapidly.

Contributing factors include westernisation, sedentary life style along with dependency on technology for most of human activates, beside availability of ready high fat, high energydense foods all contribute to the rapid growth of the problem.

Prevalence of obesity (body mass index $(\mathrm{BMI})>30$ ) in Europe is reported in the range 10$20 \%$ in men and $15-25 \%$ in women. ${ }^{[1]}$

Overweight and obesity are associated with insulin resistance, type 2 diabetes, dyslipidemia, hypertension, cardiovascular disease, gallbladder disease, and certain forms of cancer, nonalcoholic steatohepatitis, gastroesophageal reflux, obstructive sleep apnea, arthritis, gout, and polycystic ovary syndrome. ${ }^{[2]}$

Furthermore obesity impairs quality of life, and reduces life expectancy. ${ }^{[3,4]}$ 
Obesity epidemic is associated with increased burden health-care costs and more utilization of healthcare resources. ${ }^{[5]}$

Weight losses of just 5-10\% of initial body weight significantly increased the odds of achieving clinically meaningful changes in glycemic control, blood pressure, HDL cholesterol, and triglycerides at 1 year. ${ }^{[6]}$

In the Diabetes Prevention Program, it became clear that in subjects with a BMI>24 modest weight loss of only $5.6 \mathrm{~kg}$ on average reduced the incidence of diabetes by $58 \%{ }^{[7,8]}$

Bariatric surgery has proved to be the most effective mode of treatment of the morbidly obese patients and to date it is the only option resulting in substantial and durable long-term weight loss. ${ }^{[8]}$

A number of factors determine the decision for bariatric surgery based on clinical need, and the potential for long-term outcome benefits against the risks for each patient.

In this assignment the assessment of suitability of a patient for bariatric surgery and pre surgical preparation them is outlined.

The decision to refer patients for bariatric surgery is based on a number of criteria as, obesity degree, patients' age, and absence of any medical or psychological problems that increased the surgical mortality or adversely affect the outcome.

The pre surgical evaluation also involves searching for factors responsible for failure of life style modification and medical treatment in promoting weight loss, these include under lying pathology or drug induced obesity, lack of proper motivation or dietary advices from an expert personal, under lying psychological problem that may precludes the patient from coping with medical treatment and dietary advices.

\section{Degree of obesity}

According to AACE/TOS/ASMBS Guidelines suitable candidates for bariatric surgery are those patients with a BMI $>40 \mathrm{~kg} / \mathrm{m}^{2}$ without coexisting medical problems and for patients with a BMI $>35 \mathrm{~kg} / \mathrm{m}^{2}$ and 1 or more severe obesity related co-morbidities that could be improved by weight loss, including T2D, hypertension, hyperlipidemia, obstructive sleep apnea (OSA), obesity-hypoventilation syndrome (OHS), Pickwickian syndrome (a combination of OSA and OHS), non-alcoholic fatty liver disease (NAFLD) or nonalcoholic steatohepatitis (NASH), pseudotumor cerebri, gastroesophageal reflux disease (GERD), asthma, venous stasis disease, severe urinary incontinence, debilitating arthritis, or considerably impaired quality of life.(Recommendation grade A) ${ }^{[9]}$ 
The evidence for benefit for surgery in these two groups is strong (best evidence level (BEL) 1); based on meta-analysis of randomized controlled trials (MRCT) and randomized controlled trials (RCT).

Studies have demonstrated benefit on mortality, weight loss, diabetes remission; improved beta-cell function and improved pulmonary function.

According to AACE/TOS/ASMBS Guidelines patients with BMI of $30-34.9 \mathrm{~kg} / \mathrm{m}^{2}$ with diabetes or metabolic syndrome may also be offered a bariatric surgery although a welldesigned clinical trials with long follow-up periods to demonstrate safety and benefits in surgical group, compared to medical therapy comparator group is lacking. [9]

\section{Age}

The evidence for effect of age on surgical outcome is conflicting some Surveillance studies have found that older age was a predictor for increased risk of morbidity and mortality after bypass surgery. ${ }^{[10,11]}$

However, more recent studies found that advanced age was not predictor of increased mortality risk after bypass surgery. ${ }^{[12,13]}$

Expert opinion suggests poorer outcomes and more complications in older people.

Some centres have established cut-off levels for age at 65-70 years, while others primarily consider overall health risks and physiological status.

Most guidelines do not recommend the surgery for people aged less than 18 years, as it is more appropriate to address lifestyle issues within the family and social settings.

\section{History and examination with the aim of:-}

1. Rule out secondary causes of obesity should be considered, these include hypothyroidism, cushing's syndrome, growth hormone deficiency and hypothalamic pathology and medications.

Examples of drugs that can aggravate weight gain include diabeteis medications, especially sulfonylureas and thiazolidinediones and insulins, corticosteroids, oral contraceptives, tricyclics antidepressants, monoamine-oxidase inhibitors, anticonvulsants, (sodium valproate, gabapentin, vigabatrin), antipsychotics, Secondary causes of obesity sometimes are resistant to dietary measures and medication unless the underlying condition managed.

Rule out medical contraindications to bariatric surgery - for example, severe gastrointestinal disease, active cancer, severe heart failure, unstable coronary artery 
disease, advanced liver disease with portal hypertension, uncontrolled obstructive sleep apnoea with portal hypertension, end-stage lung disease and serious blood or autoimmune disorders and pregnancy.

Crohn's disease may be a relative contraindication to Roux-en-Y gastric bypass and Biliopancreatic diversion, ${ }^{[14,15]}$ and is listed by the manufacturer as a contraindication to the LAP-BAND ${ }^{\circledR}$ system. ${ }^{[16]}$

Laparoscopic surgery may be difficult or impossible in patients with giant ventral hernias, severe intra-abdominal adhesions, large liver, high BMI with central obesity or physiological intolerance of pneumoperitoneum. ${ }^{[16]}$

2. Look for comorbidities and other factors that may affect the outcome of the surgery Any obesity-related comorbidities should be managed before surgery, when they are identified, and patients may need to be referred to the appropriate specialists (for example, cardiologist, respiratory physician, and endocrinologist).

3. The following medical risk factors may require specific interventions prior to surgery:

- Optimize glycemic control in diabetic patients to achieve $\mathrm{HbA1c}<7$ in oreder to reduce adverse events

- Control blood pressure and optimize treatment for any cardiac or pulmonary disease

- In those with obstructive sleep apnoea consider pre-operative initiation of continuous positive airway pressure (CPAP) for at least 4 weeks pre-operatively to reduce hypercarbia, hypoxaemia and pulmonary artery vasoconstriction

- Hypercoagulability and thromboembolic risk is high among obese patients and all should receive thromboembolic prophylaxis.

- Patients should stop smoking at least 8 weeks before bariatric surgery and should plan to quit smoking or to participate in a smoking cessation program postoperatively

In those with high risk the decision of bariatric surgery will depend on whether the benefits outweigh the potential complications of the surgery.

\section{Psychological evaluation}

Obese patients are more likely than the non obese counterparts to have psychopological problems as somatization, social phobia, depression, generalized anxiety disorder, obsessivecompulsive disorder, substance abuse/dependency, binge-eating disorder ${ }^{[16,17]}$ 
Patients with psychiatric disorders may have a suboptimal outcome after bariatric surgery. ${ }^{[16 \text {, }}$ 17]

Pre surgical psychological evaluation is important to help avoid adverse post-operative outcomes

Surgery should be undertaken only after there has been a comprehensive evaluation of any psychosocial or other factors that may affect adherence to post-operative care requirements. Bariatric surgery should not be performed on patients who have severe untreated depression active uncontrolled schizophrenia or psychosis, binge eating disorders, heavy alcohol use or

illicit substance use disorder, severe mental retardation affecting decision-making, or ability to adhere to behavioural modification (dietary and other requirements) post-surgery

The patient's evaluation should ensure that they have realistic expectations procedure about the surgery (either in terms of weight loss or in terms of the impact on life in general), and they have the motivation and capacity to make the changes needed for a successful long-term outcome from the surgery

5. Pre-operative education and counselling, to patients and, if appropriate, members of their families is particularly important in improving the patient's understanding of the procedure and ensuring they have realistic expectations about the outcomes. Information should include details about the potential benefits of surgery, dietary and lifestyle implications and the associated risks, including complications and perioperative mortality rates. Group alone is not sufficient: patients should receive individualised preparation and education for surgery. Dietary advices and, support from a dietitian to help them change their eating habits and prevent complications from surgery. Loss of $10 \%$ of Excess body weight loss before surgery is associated with shorter hospitalization and more rapid weight loss. ${ }^{[18]}$

Nonalcoholic fatty liver disease is a risk factor for liver tearing during surgery and preoperative very-low-calorie diet for 6 weeks has been shown to reduce liver volume by $20 \%$ and to improve access to the upper stomach during laparoscopic surgery ${ }^{[19,20]}$, with $80 \%$ of the volume change occurring in the first 2 weeks. ${ }^{[19]}$

\section{Laboratory investigations}

pre-operative investigation include complete blood count, ferritin, lipid profile, thyroid function tests, blood sugar, glycated haemoglobin ( $\mathrm{Hb} \mathrm{A1c}$ ), liver function tests, urea, electrolytes and creatinine, prothrombin time (PT)/partial thromboplastin time (PTT), 
Cardiovascular evaluation includes electrocardiogram and stress ECG test if clinically indicated.

Respiratory evaluation may include chest radiograph, arterial blood gas, and pulmonary function tests.

Sleep apnea may be diagnosed by sleep study and the patient started on continuous positive airway pressure prior to surgery.

Upper endoscopy may be used if suspicion of gastric pathology exists. If H. pylori infection is present, preoperative therapy is advised. ${ }^{[16]}$

Ultrasound may be used to detect non alcoholic fatty liver, gallstones, allowing the surgeon to decide on concomitant cholecystectomy. In cases of suspected cirrhosis, biopsy may be indicated.

\section{Patient's informed consent to surgery to surgery}

The patient should be well informed about the risks and benefits, and is prepared and able to commit to dietary and lifestyle changes.

He must be able to give fully informed consent to bariatric surgery and commit to postoperative care plans.

It should reflect that: ${ }^{[21]}$

a. The patient fully understands the potential benefits, risks and long-term consequences associated with the procedure

b. The choice of surgical intervention was made jointly by the patient and the healthcare professionals responsible for treatment, following detailed individualised assessment and discussion of risks and benefits

c. The patient is motivated to make necessary dietary and lifestyle changes

d. The patient commits to long-term follow-up after surgery.

\section{Perioperative care}

The best results are obtained when the surgical team includes an expert anaesthesist support, and highly qualified nurses beside an experienced bariatric surgeon who is able to manage, complication in the intraoperative or the post-operative period.

Availability of a full spectrum of expert consultants (for example, cardiologists, respiratory physicians and psychiatrists) is also necessary.

Management plans should be individualised to ensure optimal results. 


\section{Multidisciplinary input}

For optimal results of surgery specialist care is provided by a multidisciplinary team that comprises of physicians, surgeons, dieticians and nurses, and should ensure a joint approach to the delivery of care based on the best available evidence. ${ }^{[9,22]}$

\section{Conclusion}

Obesity is one of the most important public health challenges of the present century; its prevalence is increasing rapidly. Overweight and obesity are associated increased morbidity and mortality and impaired quality of life. Weight losses is associated with improvement or even cure of cardiovascular risk factors with cardiometabolic benefit. The decision to refer patients for bariatric surgery is based on a number of criteria as, obesity degree, in addition to presence of 1 or more of the obesity related morbidities. The pre surgical evaluation also involves searching for factors responsible for failure of life style modification and medical treatment in promoting weight loss, these include under lying pathology or drug induced obesity, lack of proper motivation or dietary advices from an expert personal, under lying psychological problem that may precludes the patient from coping with medical treatment and dietary advices. Pre surgical evaluation is important to rule out any medical or psychological problems that increased the surgical mortality or adversely affect the outcome.

\section{$\underline{\text { References }}$}

1. James WPT, Rigby N, Leach R: The obesity epidemic, metabolic syndrome and future prevention strategies. Eur J Cardiovasc Prev Rehabil 2004; 11:3-8.

2. Must A, Spadano J, Coakley EH et al. The disease burden associated with overweight and obesity. JAMA.1999; 282(16):1523- 1529

3. Fontaine KR, Redden DT, Wang C et al.Years of life lost due to obesity. JAMA $.2003 ; 289(2): 187-193$

4. Allison DB, Fontaine KR, Manson JE et al. Annual deaths attributable to obesity in the United States. JAMA. 1999;282:1530-1538

5. Finkelstein EA, Trogdon JG, Cohen JW, Dietz W: Annual medical spending attributable to obesity: payer- and service-specific estimates. Health Aff (Millwood) 2009; 28:w822-w831. 
6. Wing RR, Lang W, Wadden TA, et al.; Look AHEAD Research Group. Benefits of modest weight loss in improving cardiovascular risk factors in overweight and obese individuals with type 2 diabetes. Diabetes Care 2011;34:1481-1486

7. Knowler WC, Barrett-Connor E, Fowler SE, Hamman RF,Lachin JM, Walker EA \& Nathan DM. Reduction in the incidence of type 2 diabetes with lifestyle intervention or metformin. New England Journal of Medicine. 2002; 346 393-403.

8. Bult MJ, van Dalen T, Muller AF. Surgical treatment of obesity. Eur J Endocrinol. 2008;158(2):135-45.

9. Mechanick JI, Youdim A, Jones DB, et al. Clinical Practice Guidelines for the Perioperative Nutritional, Metabolic, and Nonsurgical Support of the Bariatric Surgery Patient—2013 Update: Cosponsored by American Association of Clinical Endocrinologists, The Obesity Society, and American Society for Metabolic \& Bariatric Surgery. Endocr Pract. 2013;19(No. 2) 337-372

10. DeMaria EJ, Murr M, Byrne TK. Validation of the obesity surgery mortality risk score in a multicenter study proves it stratifies mortality risk in patients undergoing gastric bypass for morbid obesity. Ann Surg. 2007; 246:578-582.

11. DeMaria EJ, Portenier D, Wolfe L. Obesity surgery mortality risk score: Proposal for a clinically useful score to predict mortality risk in patients undergoing gastric bypass. Surg Obes Relat Dis. 2007;3:134-140.

12. Dorman RB, Abraham AA, Al-Refaie WB. Bariatric Surgery Outcomes in the Elderly: An ACS NSQIP Study. J Gastrointest Surg. 2012; 16:35-44.

13. The Longitudinal Assessment of Bariatric Surgery (LABS) Consortium. Perioperative Safety in the Longitudinal Assessment of Bariatric Surgery. N Engl J Med. 2009;361:445-454.

14. Ahn LB, Huang CS, Forse RA et al. Crohn's disease after gastric bypass surgery for morbid obesity: is there an association? Inflamm Bowel Dis. 2005;11(6):622-624

15. Pretolesi F, Camerini G, Marinari GM et al .Crohn disease obstruction of the biliopancreatic limb in a patient operated for biliopancreatic diversion for morbid obesity. Emerg Radiol. 2006;12(3):116-118

16. Society of American Gastrointestinal and Endoscopic Surgeons. Guidelines for Clinical Application of Laparoscopic Bariatric Surgery.2008; available at https://www.sages.org/publications/guidelines/guidelines-for-clinical-application-oflaparoscopic-bariatric-surgery/ 
17. Kinzl JF, Schrattenecker M, Traweger C et al. Psychosocial predictors of weight loss after bariatric surgery. Obes Surg.2006;16(12):1609-1614

18. Still CD, Benotti P, Wood GC et al. Outcomes of preoperative weight loss in high risk patients undergoing gastric bypass surgery. Arch Surg. 2007;142(10):994-998

19. Colles SL, Dixon JB, Marks P et al. Preoperative weight loss with a very-low energy diet: quantitation of changes in liver and abdominal fat by serial imaging. Am J Clin Nutr .2006;84(2): 304-311

20. Lewis MC, Phillips ML, Slavotinek JP et al. Change in liver size and fat content after treatment with Optifast very low calorie diet. Obes Surg. 2006;16(6):697-701

21. Advisory Committee on Access to Elective Surgery (ACAES) .Surgery for morbid obesity Framework for bariatric surgery in Victoria's public ospitals.2009; available at http:// www.health.vic.gov.au

22. CG189 Obesity: NICE guideline.2014; available at http:// www.nice.org.uk 